\title{
Uso de modelos lineales generalizados en el conteo de Leptopharsa gibbicarina (Hemiptera: Tingidae) en palma de aceite
}

\author{
Use of generalized linear models in the Leptopharsa gibbicarina \\ (Hemiptera: Tingidae) count in oil palm
}

\section{ELOINA MESA-FÚQUEN'; JUAN SEBASTIÁN HERNÁNDEZ-HERNÁNDEZ²; JHONATAN EDUARDO CAMPEROS ${ }^{3}$}

\begin{abstract}
M. Sc. Estadística, Corporación Centro de Investigación en Palma de Aceite, Bogotá, D. C., Colombia, emesa@cenipalma.org, https://orcid.org/0000-00031964-1767. ${ }^{2}$ Estadístico, Corporación Centro de Investigación en Palma de Aceite, Bogotá, D. C., Colombia, jhernandez@cenipalma.org, https://orcid. org/0000-0003-1758-7955. 3. Ing. Agrónomo, Corporación Centro de Investigación en Palma de Aceite, Barrancabermeja, Colombia, jcamperos@cenipalma.
\end{abstract} org, https://orcid.org/0000-0002-5133-4225.

\begin{abstract}
Autor para correspondencia
Eloisa Mesa-Fuquen. Estadística M Sc. Corporación Centro de Investigación en Palma de Aceite, Bogotá, D. C., Colombia,emesa@cenipalma.org, https://orcid.org/0000-0003-19641767.

Citación sugerida

MESA-FÚQUEN, E.; HERNÁNDEZ-HERNÁNDEZ, J. S.; CAMPEROS, J. E. 2021. Uso de modelos lineales generalizados en el conteo de Leptopharsa gibbicarina (Hemiptera: Tingidae) en palma de aceite. Revista Colombiana de Entomología 47 (1): e7661. https://doi. org/10.25100/socolen.v47i1.7661

Recibido: 15-mar-2019

Aceptado: 02-nov-2020

Publicado: 28-may-2021

Revista Colombiana de Entomología ISSN (Print): 0120-0488

ISSN (On Line): 2665-4385

https://revistacolombianaentomologia.univalle.edu.co

Open access

(c) (i) (ㅇ) BY-NC-SA 4.0
\end{abstract}

Publishers: Sociedad Colombiana de Entomología SOCOLEN (Bogotá, D. C., Colombia) https://www.socolen.org.co

Universidad del Valle (Cali, Colombia)

https://www.univalle.edu.co

(C) 2021 Sociedad Colombiana de Entomología SOCOLEN y Universidad del Valle - Univalle
Resumen: El análisis de los datos provenientes del recuento de eventos debe tener en cuenta las herramientas apropiadas para tratar este tipo de variables. Un enfoque válido para hacer frente a esta tarea consiste en el uso del Modelo Lineal Generalizado (MLG). Esto permite trabajar con funciones de densidad como Poisson o binomial negativa. Este trabajo presenta los resultados del análisis de los datos de un experimento realizado en el campo experimental de investigación de Cenipalma ubicada en el municipio de Barrancabermeja (Colombia). El experimento se estableció para evaluar, a nivel de campo, el hongo entomopatógeno Purpureocillium lilacinum como una estrategia para controlar la población de la chinche de encaje, Leptopharsa gibbicarina. El experimento se realizó en dos parcelas, en una de ellas se implementó el control de L. gibbicarina con P. lilacinum como sugería el programa de investigación de Entomología de Cenipalma y en la otra parcela no se tuvo ninguna estrategia de control. Los datos se analizaron mediante el uso de MLG y dos funciones de densidad diferentes (Poisson y binomial negativa). Los resultados mostraron diferencias estadísticamente significativas entre los tratamientos. El estimador de máxima verosimilitud asociado al tratamiento $P$. lilacinum indicó que la aplicación del hongo entomopatógeno reduce la población de L. gibbicarina con una tasa de incidencia de 0,28 veces más que el testigo.

Palabras clave: Sobredispersión, función enlace, hongo entomopatógeno, equidispersión, chinche de encaje, Leptopharsa gibbicarina.

Abstract: The analysis of data coming from the counting of events must consider tools that are appropriate to deal with this type of variable. A valid approach to cope with this task consists of using the Generalized Linear Model (GLM). The use of GLM allows working with density functions such as Poisson or Negative Binomial. This work presents the results of analyzing the data from an experiment carried out at the Research Station of Cenipalma located in the municipality of Barrancabermeja (Colombia). The experiment was established to evaluate, at the field level, the entomopathogenic fungus Purpureocillium lilacinum as a strategy to control the population of the lace bug, Leptopharsa gibbicarina. The experiment was carried out in two plots, in one of them, the control of L. gibbicarina with P. lilacinum was implemented as suggested by the Entomology research program of Cenipalma and, in the other plot there was no control strategy. The data were analyzed by using GLM and two different density functions (Poisson and the negative binomial). Results showed statistically significant differences between the treatments. The estimator of the maximum likelihood associated with the $P$. lilacinum treatment indicated that the application of the entomopathogenic fungus reduced the populations of $L$. gibbicarina with a rate of incidence of 0.28 times more than the control.

Keywords: Over dispersion, link function, entomopathogenic fungus, equidisperssion, lace bug, Leptopharsa gibbicarina.

\section{Introducción}

En algunas investigaciones se registran entre las variables de interés el recuento de eventos, es decir el conteo de un evento de interés que ocurre en un tiempo o espacio dado; en algunas disciplinas estas variables se analizan utilizando el Modelo Lineal General (ML) con el propósito de expresar la relación entre la variable de respuesta llamada independiente y una o más variables explicativas. Este modelo asume linealidad, normalidad, homogeneidad en las varianzas e independencia en los errores 
aleatorios, (Draper y Smith 1981). Con el objeto de lograr que estos supuestos se cumplan se recurre a transformaciones de los datos para lograr la homogeneidad de varianzas (Kuehl 2000; Melo Martínez et al. 2007). Cuando no se logra la normalidad, se acude a la estadística no paramétrica para realizar el análisis (Zar 1996; Lehmann 2006). La dificultad con las transformaciones se da en términos de la interpretación de los resultados los cuales no corresponden a la escala inicial de medición de la variable de respuesta.

Los Modelos Lineales Generalizados (MLG), son una extensión del ML, para modelar variables que pertenezcan a la familia de distribución de probabilidad exponencial (Nelder y Wedderburn 1972); esta familia contiene las distribuciones: normal, binomial, Poisson y binomial negativa, entre otras. El MLG propuesto, en el presente estudio, está conformado por: i) una componente aleatoria dada por la variable de respuesta $Y_{i}$ y su distribución de probabilidad; ii) una componente sistemática conformada por las variables explicativas $X_{i}$ expresadas como una función lineal conocida como el predictor lineal $\eta_{i}$; iii) una función de enlace denotada por $\mathrm{g}$ (.) que relaciona el valor esperado de la variable de respuesta $\mu_{i}$ con las variables explicativas $X_{\mathrm{i}}$ de la siguiente manera: $g\left(\mu_{i}\right)=\beta_{0}+\beta_{1} x_{\mathrm{i} 1}+\ldots$, $+\beta_{p} x_{i p}$ para $i=1,2, \ldots, n$; las $\mathrm{p}$ variables del lado derecho de la expresión corresponden a las variables explicativas y pueden ser factores de estudio, covariables e interacciones entre estas. Los parámetros $\beta_{0}, \beta_{1}, \ldots, \beta_{p}$ son estimados utilizando máxima verosimilitud. La diferencia entre el ML y el MLG está en que este último incluye una función de enlace $g$ (.) que relaciona la componente aleatoria y la componente sistemática de tal manera que $g\left(\mu_{i}\right)=\beta_{0}+\beta_{1} x_{i 1}+\ldots+\beta_{p} x_{i p}$ y no como en el ML que $\mu_{i}=\beta_{0}+\beta_{1} x_{i 1}+\ldots+\beta_{p} x_{i p}$; de esta forma, la función de enlace lo que garantiza es que los valores estimados tomen valores en la misma escala de los esperados de la variable de respuesta, lo que no ocurre en el ML, en el cual los valores estimados pueden tener valores para $\mu_{i}<0$.

Este artículo tiene como propósito describir y aplicar el Modelo Lineal Generalizado (MLG) para el análisis de datos de un ensayo que se realizó en el Campo Experimental El Palmar de la Vizcaína (CEPV) de Cenipalma, dirigido a validar el control de Leptopharsa gibbicarina (Froeschner, 1976) (Hemiptera: Tingidae) a través del uso del hongo entomopatógeno Purpureocillium lilacinum (Thom.).

\section{Materiales y métodos}

El MLG, propuesto por Nelder y Wedderburn (1972) es una extensión del ML, en el cual la variable de respuesta puede tener una distribución normal, binomial, Poisson o binomial negativa entre otras. El MLG está conformado por tres componentes: aleatoria, sistemática y una función de enlace; la componente aleatoria se refiere a los valores observados de la variable de respuesta con función de distribución $\mathrm{f}$ (y) la cual debe pertenecer a la familia de funciones exponenciales. La componente sistemática $\theta_{i}=x_{i} \beta$ corresponde a la variabilidad de la variable de respuesta $\mathrm{Y}$ que es explicada por $\mathrm{X}$ a partir de una combinación lineal $x_{i} \beta$, conocida como el predictor o conjunto de parámetros y variables explicativas; la función de enlace relaciona las componentes aleatoria y sistemática, y se expresa como $g\left(\mu_{i}\right)$. La importancia de esta función es que permite transformar los valores esperados a la misma escala de los del predictor, es decir $g\left(\mu_{i}\right)=x_{i} \beta$; por ejemplo, en el caso particular de la distribución de Poisson, se espera que $\mu_{i}$ tome valores $1,2,3, \ldots$, al igual que los estimados, sin embargo estos últimos pueden tomar valores negativos cuando no se considera la función de enlace como en el caso del ML.

Si la variable de respuesta de interés es cuantitativa y discreta y se registra como el conteo de un evento en un espacio o tiempo dados, puede hacerse uso de la distribución Poisson (Agresti 2002). Esta distribución se expresa de la forma $\mathrm{P}(Y=y)=\frac{e^{-\mu} \mu^{y}}{y !}$, para $y=0,1,2, \ldots$ tiene un solo parámetro $\mathrm{y} \mathrm{E}[\mathrm{y}]=\mu=\operatorname{Var}[\mathrm{y}]$. La distribución pertenece a la familia de distribuciones exponenciales y se expresa de la forma $f(y ; \mu)=\exp [-\mu]\left[\frac{1}{y !}\right] \exp [y \log (\mu)]$, haciendo las siguientes sustituciones: $a(\theta)=\exp (-\mu) ; b(y)=\frac{1}{\mathrm{y} !} ; \mathrm{y} Q(\theta)=y \log (\mu)$ (Agresti 2002).

El uso del MLG sigue un conjunto de pasos al igual que el ML para el análisis de los datos: i) identificación y selección del modelo: se evalúan los supuestos de la componente aleatoria, se establece la componente sistemática de acuerdo con la forma como se observaron o registraron los datos y se determina la función de enlace; se debe considerar la significancia o no de cada uno de los términos del modelo y la interpretación de los mismos (Lindsey 1995). Los modelos propuestos deben evaluarse en términos de la magnitud de la discrepancia entre los datos observados y los valores esperados, la cual se formula acorde a la distribución de la componente aleatoria. ii) Estimación de parámetros: se hace a partir de métodos iterativos para ecuaciones no lineales como las generadas por este tipo de modelos (Nelder y Wedderburn 1972), a partir de la resolución de estas ecuaciones se determina el máximo valor (Dobson y Barnett 2018); iii) Evaluación del modelo: esta se centra en la propuesta de las componentes del MLG: distribución de la componente aleatoria, especificación de las variables explicativas o componente sistemática y selección de la función de enlace asociada a la componente aleatoria (Agresti 2002); una especificación incorrecta de la componente sistemática genera errores que pueden generar violación de supuestos en la distribución de la variable de respuesta, por ejemplo, en el caso de la distribución de Poisson la relación de igualdad entre la media y la varianza, conocida como equidispersión (Agresti 2002; Morales y López 2009; Torres Blanco 2011). Cuando este supuesto no se cumple, la media de la variable de respuesta puede estar cambiando y esta variación debe ser modelada. Si esto ocurre y, la variable de respuesta de interés $Y \sim$ $P(\lambda)$, entonces el parámetro $\lambda$ sigue una distribución Gamma, es decir $\lambda \sim G(k, \mu)$, y su expresión es de la forma

$$
f(\lambda ; k, \mu)=\left(\left(\frac{\left(\frac{k}{\mu}\right)^{k}}{\Gamma(k)}\right) \exp \left(\frac{-k \lambda}{\mu}\right) \lambda^{k-1} \operatorname{con} \lambda \geq 0 ;\right.
$$

esta distribución tiene $E(\lambda)=\mu y \quad V(\lambda)=\frac{\mu^{2}}{k}$. (Agresti 2002); la mezcla de estas dos distribuciones (Poisson y Gamma) genera la distribución Binomial Negativa (BN), que es la de interés, dado que permite modelar conteos en casos de sobredispersión (Agresti 2002).La distribución BN se expresa de la siguiente forma:

$$
P(y ; \mu, k)=\left(\frac{\Gamma(y+k)}{\Gamma(k) \Gamma(y+1)}\right)\left(\frac{k}{(\mu+k)^{k}}\right)\left(1-\left(\frac{k}{(\mu+k)}\right)\right)^{y}
$$

su esperanza y varianza están dadas por $\mu \mathrm{y} \mu+\frac{\mu^{2}}{k}$, respectivamente (Agresti 2002); el parámetro de dispersión es $k^{-1}$, el cual a medida que aumenta indica que la sobredispersión es mayor en el modelo de Poisson.

Para mostrar el uso del MLG, se utilizaron datos generados en un ensayo, llevado a cabo en Cenipalma, para validar el control del hemíptero Leptopharsa gibbicarina, responsable de ocasionar lesiones en la hoja de la palma de aceite, al 
facilitar el desarrollo del hongo Pestalotiopsis palmarum. Esta especie genera necrosis, destruye la lámina foliar y disminuye así la capacidad fotosintética de la planta. Esta enfermedad se denomina Pestalotiopsis y afecta la producción. En Cenipalma se encontró que la aplicación del hongo entomopatógeno Purpureocillium lilacinum (CPP10601) en dosis de $1 \times 10^{13}$ conidias/ha, controla el estado adulto de L. gibbicarina (Barrios et al. 2016).

Con el propósito de aplicar el MLG, se utilizaron los datos registrados en un ensayo en el Campo Experimental El Palmar de La Vizcaína, realizado en un lote de 3,9 Has, ubicado en la vereda Peroles, de Barrancabermeja, Santander, Colombia (6 $\left.6^{\circ} 59^{\prime} 32^{\prime}{ }^{\prime} \mathrm{N} 73^{\circ} 43^{\prime} 04^{\prime \prime} \mathrm{O}\right)$, con presencia de pestalotiopsis y de L. gibbicarina. El lote se dividió en dos áreas de similar tamaño y se asignó aleatoriamente a una de las áreas la aplicación de P. lilacinum y a la otra no se le hizo aplicación, siendo el testigo. La aplicación del entomopatógeno se realizó en abril de 2016 en el híbrido OxG cultivar Coarí x LaMé, año de siembra 2012, de acuerdo con los criterios y especificaciones indicados en Barrios et al. (2016). En cada una de las áreas se hizo el conteo inicial de adultos de L. gibbicarina en la hoja 25 (según filotaxia de la palma) utilizando muestreo sistemático descrito por Särndal et al. (2003); se seleccionó aleatoriamente una palma como punto de partida y, seguidamente, cada dos palmas se realizó el conteo de insectos hasta recorrer todas las palmas de cada área; además se aplicó el entomopatógeno en el área seleccionada. A los 30 días después de la aplicación (30 dda) del hongo, se realizó el conteo de insectos para las dos áreas, en la misma palma y hoja evaluada al inicio. Los datos se analizaron con el uso del software estadístico SAS Institute Inc (2008).

\section{Resultados}

Descripción de los datos. En la Tabla 1 se registran estadísticas descriptivas para el tratamiento control y para el tratamiento donde se aplicó Purpureocillium lilacinum; el conteo de L. gibbicarina para los dos tratamientos antes de aplicar el hongo presenta diferencias considerables en sus promedios y mayor variabilidad en el tratamiento de validación, situación que se presentó por la dispersión del insecto y que se encontró después de aleatorizar el área de aplicación de los tratamientos. La evaluación a los 30 días después de aplicar el hongo presenta menores diferencias en términos de promedio.

Análisis de los datos utilizando el MLG. Para el análisis se utilizó la distribución de Poisson con un factor de estudio (tratamientos) y se consideró la lectura antes de aplicar los tratamientos como una covariable (Arango Londoño et al. 2009). Se utilizó como función de enlace $g\left(\mu_{i}\right)=\log \left(\mu_{i}\right)$. En la Tabla 2 se muestran resultados del análisis que permiten verificar la bondad de ajuste del modelo propuesto.

Tabla 1. Medias y varianzas para conteos de Leptopharsa gibbicarina (Hemiptera: Tingidae) registrados antes y después de la aplicación del hongo entomopatógeno Purpureocillium lilacinum.

\begin{tabular}{cccccccc}
\hline & \multicolumn{4}{c}{ Control } & \multicolumn{3}{c}{ Validación } \\
\hline Variable & $\mathbf{n}$ & Media & Varianza & $\mathbf{n}$ & Media & Varianza \\
\hline dda0 & 13 & 25,23 & 745,69 & 14 & 89,21 & 6460,64 \\
dda30 & 13 & 69,15 & 11139,42 & 14 & 87,78 & 4044,64 \\
\hline
\end{tabular}

dda: días después de la aplicación.
Tabla 2. Criterios para evaluar la bondad de ajuste del modelo de distribución de Poisson.

\begin{tabular}{lccc}
\hline \multicolumn{1}{c}{ Criterios } & GL & Valor & Valor/GL \\
\hline Desviación & 50 & 3236,85 & 64,74 \\
Desviación escalada & 50 & 3236,85 & 64,74 \\
$\chi^{2}$ de Pearson & 50 & 3815,21 & 76,30 \\
Log de verosimilitud & & 12296,67 & \\
Log de verosimilitud total & & $-1761,72$ & \\
AIC (más pequeño mejor) & & 3531,43 & \\
AICC (más pequeño mejor) & & 3532,25 & \\
BIC (más pequeño mejor) & & 3539,39 & \\
\hline
\end{tabular}

A partir de la desviación se evalúa primero la equidispersión del modelo, es decir, si la media y la varianza del modelo son iguales como debe ocurrir en el modelo de Poisson. El valor de la desviación para este caso fue de 64,41 que es mucho mayor de 1,0 indicando que esta afirmación no se cumple (McCullagh y Nelder 1989), es decir el modelo presenta sobredispersión, la varianza es mayor que la media para los datos analizados, situación que se presenta con cierta frecuencia en la distribución de Poisson (Hinde y Demétrio 1998; Agresti 2002).

Una alternativa para el análisis de los datos considerada en este trabajo fue analizarlos a través de la regresión binomial negativa que asume la varianza como una función de la media (Navarro et al. 2001; Morales y López 2009). La bondad de ajuste de este modelo se muestra en la Tabla 3.

Los resultados muestran un efecto importante de los tratamientos con $P=0,0019$, indicando diferencias significativas entre los dos tratamientos; no se observa efecto de la covariable con $\mathrm{P}=0,9674$ (Tabla 4). La estimación del parámetro de dispersión es de 1,084 con un intervalo de confianza del $95 \%$ de $(0,764 ; 1,537)$; este intervalo no contiene el cero, luego el parámetro $>0$, indicando que el modelo Binomial Negativo es más apropiado que el de Poisson para modelar los datos. El estimador para el tratamiento 1 (aplicación del hongo) fue de $e^{-1,263}=0,2828$ e indica la diferencia esperada entre este tratamiento y el tratamiento control, así indica que la tasa de incidencia del tratamiento 1 (aplicación del hongo) es 0,2828 veces mayor que el testigo.

Tabla 3. Criterios para evaluar la bondad de ajuste del modelo de regresión con la binomial negativa.

\begin{tabular}{lccc}
\hline \multicolumn{1}{c}{ Criterios } & GL & Valor & Valor/GL \\
\hline Desviación & 50 & 62,65 & 1,25 \\
Desviación escalada & 50 & 62,65 & 1,25 \\
$\chi^{2}$ de Pearson & 50 & 53,49 & 1,07 \\
Log de verosimilitud & & 13781,34 & \\
Log de verosimilitud total & & $-277,05$ & \\
AIC (más pequeño mejor) & & 564,09 & \\
AICC (más pequeño mejor) & & 565,34 & \\
BIC (más pequeño mejor) & & 574,04 & \\
\hline
\end{tabular}


Tabla 4. Análisis para el conteo de insectos a los 30 días de aplicación del hongo entomopatógeno a partir de la distribución binomial negativa.

Estimación de parámetros de máxima verosimilitud

\begin{tabular}{|c|c|c|c|c|c|c|c|c|}
\hline \multirow{2}{*}{$\begin{array}{c}\text { Parámetro } \\
\text { Intercepto }\end{array}$} & & \multirow{2}{*}{$\frac{\mathbf{G L}}{1}$} & \multirow{2}{*}{$\begin{array}{c}\text { Estimador } \\
4,491\end{array}$} & \multirow{2}{*}{$\begin{array}{c}\begin{array}{c}\text { Error } \\
\text { estándar }\end{array} \\
0,279\end{array}$} & \multicolumn{2}{|c|}{$\begin{array}{c}\text { Wald } 95 \% \\
\text { Límites de confianza }\end{array}$} & \multirow{2}{*}{$\begin{array}{c}\text { Wald } \\
\chi^{2} \\
257,85\end{array}$} & \multirow{2}{*}{$\begin{array}{r}\operatorname{Pr}>\chi^{2} \\
<0001\end{array}$} \\
\hline & & & & & 3,943 & 5,039 & & \\
\hline Covariable & & 1 & $-0,016$ & 0,395 & $-0,791$ & 0,759 & 0,00 & 0,9674 \\
\hline Tratamiento & 1 & 1 & $-1,263$ & 0,406 & $-2,058$ & $-0,468$ & 9,69 & 0,0019 \\
\hline Covariab*trat & 1 & 1 & 1,031 & 0,572 & $-0,090$ & 2,152 & 3,25 & 0,0715 \\
\hline Dispersión & & 1 & 1,084 & 0,193 & 0,764 & 1,537 & & \\
\hline
\end{tabular}

\section{Discusión}

En el análisis de regresión de Poisson antes de interpretar y concluir acerca de la hipótesis de interés (efecto de la aplicación el hongo entomopatógeno), es necesario verificar si el modelo propuesto es el indicado para la modelación de los datos; uno de los supuestos para este modelo es la equidispersión, es decir la media y la varianza deben ser iguales; la prueba muestra que este supuesto no se cumple indicando sobredispersión (Var $(\mathrm{Y})>\mathrm{E}(\mathrm{Y})$, Tabla 2); este resultado se esperaba dada la descripción del recuento de insectos antes y después de la aplicación del tratamiento la cual muestra relaciones varianza-promedio distantes de ser iguales para las dos mediciones (Tabla 1). La sobredispersión puede presentarse por alta variabilidad entre las unidades observadas y no considerar una estrategia de agrupación de estas con condiciones de variación similares, aunque esta situación se presenta con cierta frecuencia en el caso de la distribución de Poisson (Morales y López 2009). Una alternativa para solucionar esta situación es hacer uso de la distribución binomial negativa, la cual asume que la varianza presenta un valor superior a la media y se utilizó en este trabajo para completar el análisis de los datos utilizando MLG.

La Tabla 3 muestra el análisis de los datos utilizando la regresión binomial negativa; el estadístico chi cuadrado de Pearson no evidencia rechazar la hipótesis del modelo binomial negativo para la modelación de los datos, lo que permite su uso para el análisis estos; esta distribución ha sido utilizada en experimentos que impliquen conteo de insectos (Silva et al. 2016). La comparación de los dos tratamientos a través del estadístico $\chi^{2}$ (Tabla 4), muestra diferencia entre los dos tratamientos y eł coeficiente negativo para el tratamiento aplicación de $P$. lilacinum, indica que la aplicación de este hongo entomopatógeno genera una reducción en la población de $L$. gibbicarina, resultado acorde con lo encontrado por Barrios et al. (2016), quienes evaluaron la misma cepa de P. lilacinum en ecosistemas palmeros para el control de L. gibbicarina.

No se observó efecto de la covariable (con un $\alpha=0,05$ ) para las condiciones del lote donde se realizó la evaluación; estos aspectos admiten una recomendación confiable del hongo a nivel comercial. Sin embargo, debido a las diferentes condiciones climáticas presentes dentro de las zonas palmeras en Colombia y teniendo en cuenta la influencia que tienen factores climáticos como la temperatura, humedad relativa y radicación UV en la eficacia de los hongos entomopatógenos (Pucheta Díaz et al. 2006; Carrillo-Rayas y Blanco-Labra 2009), se sugiere realizar otros ensayos de validación bajo diferentes condiciones ambientales. Además, se recomienda: i) verificar antes de iniciar el ensayo la distribución espacial del insecto; ii) contar con repeticiones para cada uno de los tratamientos, que permita hacer inferencia acerca de la bondad del hongo para el control del insecto y finalmente iii) evaluar la posible migración de insectos de un lote al otro dada la cercanía de los mismos en el ensayo realizado.

\section{Agradecimientos}

Los autores agradecen al Fondo de Fomento Palmero administrado por Fedepalma por financiar esta investigación, así mismo, a los revisores del trabajo por sus comentarios y valiosos aportes que lo fortalecieron y a Carlos Enrique Barrios Trilleras, por sus oportunos comentarios.

\section{Literatura citada}

AGRESTI, A. 2002. Categorical Data Analysis. 2nd Edition. Jhon Wiley \& Sons, Inc., Hoboken. New Jersey, EE. UU. 710 p.

ARANGO LONDOÑO, D.; ORTEGA LENIS, D.; OLAYA OCHOA, J. 2009. Modelación del número de homicidios vía regresión de Poisson. Heurística 16: 81-90. https://bibliotecadigital.univalle. edu.co/bitstream/handle/10893/6105/Heuristica16-A07.pdf

BARRIOS, T. C. E.; BUSTILLO P., A. E.; OCAMPO R., K. L.; REINA C., M. A.; ALVARADO M., H. L. 2016. Eficacia de hongos entomopatógenos en el control de Leptopharsa gibbicarina (Hemiptera: Tingidae) en palma de aceite. Revista Colombiana de Entomología 42 (1): 22-27. https://doi.org/10.25100/socolen. v42i1.6665

CARRILLO-RAYAS, M. T.; BLANCO-LABRA, A. 2009. Potencial $\mathrm{y}$ algunos de los mecanismos de acción de los hongos entomopatógenos para el control de insectos plaga. Acta Universitaria 19 (2): 40-49. https://doi.org/10.15174/au.2009.102

DOBSON, A. J.; BARNETT, A. G. 2018. An introduction to generalized linear models. Chapman and Hall / CRC Press. 4th Edition. Boca Raton, EE. UU. 392 p.

DRAPER, N. R.; SMITH, H. 1981. Applied regression analysis. 2nd Edition. John Wiley \& Sons. New York, EE. UU. 709 p.

HINDE, J.; DEMÉTRIO, C. G. B. 1998. Overdispersion: Models and estimation. Computational Statistics \& Data Analysis 27 (2): 151-170. https://doi.org/10.1016/S0167-9473(98)00007-3

KUEHL, R. O. 2000. Design of experiments: Statistical principles of research design and analysis. 2nd Edition. Duxbury/Thomson Learning. Belmont, California, EE. UU. 666 p.

LEHMANN, E. L. 2006. Nonparametrics: Statistical methods based on ranks. Springer Science \& Business Media. New York, EE. UU. 463 p.

LINDSEY, J. K. 1995. Modelling frequency and count data. Oxford Science Publications/Clarendon Press. Oxford, England. 291 p. 
McCULLAGH, P.; NELDER, J. A. 1989. Generalized linear models. 2nd Edition. Chapman and Hall. Londres, England. 526 p. http:// www.utstat.toronto.edu/ brunner/oldclass/2201s11/readings/ glmbook.pdf

MELO MARTÍNEZ, O. O.; LÓPEZ PÉREZ, L. A.; MELO MARTÍNEZ, S. E. 2007. Diseño de experimentos: métodos y aplicaciones. Universidad Nacional de Colombia. Bogotá, Colombia. 699 p. https://www.uneditorial.com/bw-diseno-de-experimentos-metodos-y-aplicaciones-fisica.html

MORALES, M. A.; LÓPEZ, L. A. 2009. Estudio de homogeneidad de la dispersión en diseño a una vía de clasificación para datos de proporciones y conteos. Revista Colombiana de Estadística 32 (1): 59-78. https://revistas.unal.edu.co/index.php/estad/article/ view/29754

NAVARRO, A.; UTZET, F.; PUIG, P.; CAMINAL, J.; MARTÍN, M. 2001. La distribución binomial negativa frente a la de Poisson en el análisis de fenómenos recurrentes. Gaceta Sanitaria 15 (5): 447-452. https://doi.org/10.1016/S0213-9111(01)71599-3

NELDER, J. A.; WEDDERBURN, R. W. M. 1972. Generalized linear models. Journal of the Royal Statistical Society, Series A 135 (3): 370-384. https://doi.org/10.2307/2344614

PUCHETA DÍAZ, M.; FLORES MACÍAS, A.; RODRÍGUEZ NAVARRO, S.; DE LA TORRE, M. 2006. Mecanismo de acción de los hongos entomopatógenos. Interciencia 31 (12): 856-860. Disponible en: https://www.researchgate.net/publication/46406133_Mecanismo_de_accion_de_los_hongos_entomopatogenos [Fecha revisión: $1 \overline{5}$ diciembre 2020$]$

SÄRNDAL, C-E.; SWENSSON, B.; WRETMAN, J. 2003. Model assisted survey sampling. Springer Science \& Business Media. Örebro, Sweden. 694 p.

SAS INSTITUTE INC. 2008. SAS/STAT ${ }^{\circledR}$ 9.2. User's guide, Introduction to Statistical Modeling with SAS/STAT Software. SAS Institute. Cary, North Carolina, EE. UU. 60 p. https://support. sas.com/documentation/cdl/en/statugstatmodel/61751/PDF/default/statugstatmodel.pdf
SILVA, A. S.; MOTA, T. A.; PIÑEYRO, N. G.; FERNANDES, M. G.; PEREIRA, F. F. 2016. Distribución espacial de Vatiga spp. (Hemiptera: Tingidae) en el cultivo de yuca. Acta Biológica Colombiana 21 (1): 195-200. https://doi.org/10.15446/abc. v2 $1 \mathrm{n} 1.46762$

TORRES BLANCO, A. M. 2011. Estudio en bloques completos vía regresión Poisson en presencia de sobredispersión. Tesis Magister en Ciencias Estadística. Universidad Nacional de Colombia, Bogotá, Colombia. 68 p. https://repositorio.unal.edu. $\mathrm{co} / \mathrm{bitstream} / \mathrm{handle} / \mathrm{unal} / 75303 /$ anamariatorresb.2011.pdf?sequence $=1 \&$ isAllowed $=\mathrm{y}$

ZAR, J. H. 1996. Biostatistical analysis. 3th Edition. Prentice Hall. New Jersey, EE. UU. 662 p.

\section{Origen y financiación}

La presente investigación forma parte del trabajo de validación en campo del uso del hongo entomopatógeno Purpureocillium lilacinum (CPPl0601) para el control de adultos de Leptopharsa gibbicarina como paso previo a la transferencia de esta propuesta a los palmicultores colombianos. La investigación fue financiada por el Fondo de Fomento Palmero.

\section{Contribución de los autores}

Eloina Mesa-Fuquen, fue responsable de la metodología del análisis de datos, interpretación de resultados, discusión y elaboración del documento.

Juan Sebastián Hernández-Hernández, realizó la revisión de literatura, análisis de los datos e interpretación de los resultados y revisión del documento.

Jhonatan Eduardo Camperos, realizó el montaje del ensayo en campo, la captura de los datos, el análisis descriptivo y revisión del documento. 